\title{
Dynamic properties of two control systems underlying visually guided turning in house-flies
}

\author{
Martin Egelhaaf \\ Max-Planck-Institut für biologische Kybernetik, Spemannstrasse 38, D-7400 Tübingen, \\ Federal Republic of Germany \\ Accepted June 14, 1987
}

\begin{abstract}
Summary. The compensatory optomotor turning reaction as well as the turning response towards objects play an important role in visual orientation. On the basis of behavioural experiments under precisely defined stimulus conditions it is concluded that in female house-flies these motiondependent responses are mediated by two parallel control systems with different dynamic and spatial integration properties. One of them ('large-field system') is most sensitive to the motion of large textured patterns and controls the yaw torque mainly at low oscillation frequencies (below $0.1 \mathrm{~Hz}$ ) of the stimulus panorama. In contrast, the other control system ('small-field system') is tuned to the detection of relatively small moving patterns and shows its strongest responses at high oscillation frequencies (between 1 and $4 \mathrm{~Hz}$ ), i.e. in a frequency range where the large-field system contributes to the turning response with only a relatively small gain.

In free flight, house-flies do not curve smoothly but in sequences of rapid turns which induce retinal large-field motion of continually changing sign (Wagner 1986b). The dynamic properties of the large-field system might thus be interpreted as a simple strategy to almost eliminate the unwanted optomotor yaw torque induced by active self-motion. In contrast, the small-field system might still be operational under these conditions.
\end{abstract}

\section{Introduction}

Two principally different motion-dependent responses contribute to visual orientation of the fly. i) The compensatory optomotor turning reaction transforms visually perceived motion into flight torque and stabilizes the flight course against internal and external disturbances. ii) The orientation response towards objects displaces the images of objects into the frontal part of the visual field and can be induced by local retinal image motion.

As a precondition for efficient orientation towards objects in natural surroundings two substantial problems need to be overcome by the animal. i) The object has to be discriminated from its background. This figure-ground discrimination task can only be accomplished, if object and background differ in at least one respect such as in texture, contrast, luminance, colour, or retinal velocity. ii) The fly has to cope with the consequences of its visual input due to active self-motion. Since any turn in front of a contrasted background inevitably leads to rotational large-field motion in the opposite direction, the compensatory optomotor response might otherwise interfere with the orientation towards objects.

There is now good evidence that in the fly the compensatory optomotor reaction and the orientation towards objects are mediated by two parallel control systems with different sensitivities to the angular extent of the moving pattern (Geiger and Nässel 1982; Götz 1983; Heisenberg and Wolf 1984; Egelhaaf 1985a-c; Bausenwein et al. 1986). One of them ('large-field system') is most sensitive to motion of extended textured patterns. In contrast, the other control system ("small-field system') is tuned to the detection of small objects moving relative to the retina. These control systems have been attributed at the neuronal level to two different functional classes of output cells of the optic lobes. While the 'Horizontal Cells' mainly mediate information on large-field motion to the motor output (Hausen 1981; Hausen and Wehrhahn 1983; Reichardt et al. 1983; Wehrhahn 
1985). ii) The 'Figure Detection (FD) Cells' are tuned to detect small horizontally moving objects (Egelhaaf 1985b).

The role of these control systems in accomplishing figure-ground discrimination has been analysed in great detail during the last years (Reichardt et al. 1983; Egelhaaf 1985a-c). In the present study it is shown that the large-field and the small-field system have different dynamic response properties. Under natural conditions these properties allow the fly to cope with the retinal large-field image displacements due to active selfmotion, which would otherwise impede the orientation towards objects.

\section{Material and methods}

The experiments were carried out with wild type female houseflies. Musca domestica (L.), obtained from the laboratory stocks.

The flies were prepared as described by Fermi and Reichardt (1963). The head of the animal was fixed to the thorax with a mixture of wax and colophonium under light carbon dioxide anesthesia. A triangular piece of cardboard was glued to the wax just above the frontal part of the thorax. The ocelli were covered with the same mixture of colophonium and wax.

Test flies were suspended from a torque compensator which prevented both rotatory and translatory movements of the animal. It allowed direct measurement of the instantaneous yaw torque generated by the fly (e.g. Fermi and Reichardt 1963; Götz 1964). The torque response was directly inspected on the oscilloscope screen, stored on magnetic tape (3968A Instrumentation Tape Recorder, Hewlett Packard), further processed by a signal averager (4202 Princeton Applied) and finally plotted with a X-Y recorder (7047A Hewlett Packard).

The animals were positioned in the centre of two cylindrical patterns with diameters of $105 \mathrm{~mm}$ and $100 \mathrm{~mm}$, respectively. The angular extent of the outer cylinder was $360^{\circ}$ horizontally. The inner stimulus pattern consisted of a cylinder segment of $10^{\circ}$ width. The height of both cylinders amounted to $76 \mathrm{~mm}$ which corresponds, in the vertical direction, to an angular extent of the stimulus of about $\pm 21^{\circ}$ as seen by the fly. Both the background cylinder and the segment of the inner cylinder ('figure') were covered with a vertical sinusoidal grating. Its spatial wavelength was $10^{\circ}$. The two cylinders were illuminated from behind by three direct current driven fluorescent ring bulbs. The average luminance of the figure was about $185 \mathrm{~cd} / \mathrm{m}^{2}$ and that of the background was about $430 \mathrm{~cd} / \mathrm{m}^{2}$. The contrast amounted to 0.45 and 0.6 for figure and ground, respectively.

The fly was alternately stimulated by synchronous sinusoidal oscillation of the figure and its background (large-field motion) and by the figure oscillating alone while the ground was kept stationary (small-field motion). There was no interval between small-field and large-field motion. The figure was positioned in these experiments in front of the right eye at a mean angular position of $30^{\circ}$ as measured from the frontal midline of the animal. While the oscillation amplitude was kept constant $\left(20^{\circ}\right)$ in the experiments presented here, the oscillation frequency was varied over a range of almost three decades (between $0.0156 \mathrm{~Hz}$ and $8 \mathrm{~Hz}$ ). The experiments were performed under open-loop conditions, i.e. the yaw torque generated by the fly did not affect the visual stimulus.

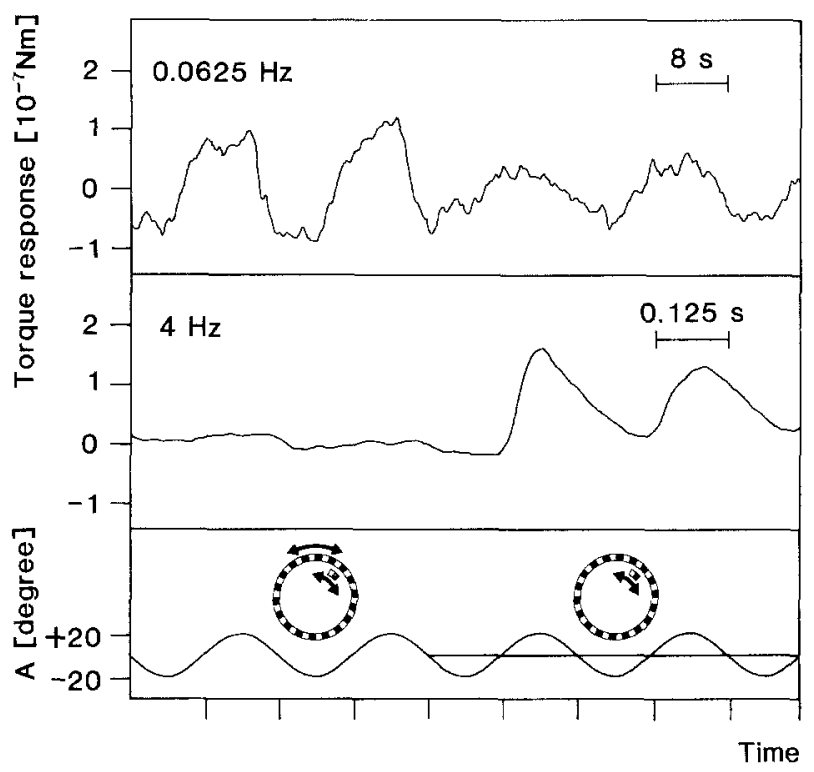

Fig. 1. Torque responses of a female Musca to large-field and small-field motion at two different oscillation frequencies. During large-field motion the pattern cylinder ('ground') was oscillated synchronously with a cylinder segment ('figure') of $10^{\circ}$ angular width. During small-field motion the figure was oscillated in front of the ground which was kept stationary. Figure and ground were covered with a vertical sinusoidal grating with a spatial wavelength of $10^{\circ}$. The mean position of the figure was in front of the right eye at $30^{\circ}$ as measured from the frontal midline. The stimulus conditions are indicated in the bottom traces which denote the deviation of figure and ground from their mean positions. Upward and downward deflections indicate clockwise and counterclockwise motion, respectively. After two cycles of synchronous oscillation of figure and ground with an amplitude of $20^{\circ}$ the ground stopped moving while the figure continued oscillating for another two cycles. The response profiles are averages obtained from several $(8$ at $0.0625 \mathrm{~Hz}$ and 64 at $4 \mathrm{~Hz}$ ) consecutive presentations of the stimulation programme. While at $0.0625 \mathrm{~Hz}$ the response to large-field motion is larger than the response to small-field motion, the response to small-field motion has a considerably larger amplitude at $4 \mathrm{~Hz}$ as compared with the response to large-field motion

\section{Results}

The experiments to be described here were aimed to analyse whether the yaw torque underlying the turning responses of female house-flies to largefield and small-field motion depends in a different way on the oscillation frequency of the stimulus pattern. Figure 1 shows examples of the instantaneous time-dependent torque responses of a fly. They were obtained at two oscillation frequencies which differed by a factor of $64(0.0625 \mathrm{~Hz}$ : upper diagram; $4 \mathrm{~Hz}$ : middle diagram). At both oscillation frequencies figure and ground were moved synchronously for the first two cycles. Then the ground stopped moving, while the figure continued oscillating for another two cycles (see bottom traces in Fig. 1). The response profiles are averages 
obtained from several $(8$ at $0.0625 \mathrm{~Hz}$ and 64 at $4 \mathrm{~Hz}$ ) consecutive presentations of the stimulation programme.

When figure and ground oscillate together, the fly tries to follow the pattern motion irrespective of the oscillation frequency; the yaw torque is then assumed to be symmetrical around zero (Fig. 1). This would reduce the relative velocity between the stimulus and the eye, if the fly were not kept stationary in space by the torque compensator. When only the figure oscillates in front of the right eye, the yaw torque signal no longer oscillates around zero. Instead, it is in the direction which would under closed-loop conditions bring the target to the front of the eye. This is most obvious at high oscillation frequencies, when the response to small-field motion is particularly large, but much less pronounced at low oscillation frequencies. As in the low-frequency example in Fig. 1, this effect may even disappear. This, however, is not a general feature of the results and might be due to uncertainties in determining the exact zero level of the fly's torque response.

Two differences between the torque responses at the low and the high oscillation frequency are particularly striking (see Fig. 1). i) At $0.0625 \mathrm{~Hz}$ the response to large-field motion is much larger than at $4 \mathrm{~Hz}$ where the response can hardly be discerned. ii) At the low oscillation frequency, the response to large-field motion is significantly larger than the response to small-field motion, but the situation is just the opposite at the high oscillation frequency. Here the response to small-field motion has a much larger amplitude than to large-field motion. It can have the same size as the response to synchronous motion of figure and ground at low oscillation frequencies (Fig. 1), or it can be even larger.

Average amplitudes of the yaw torque response to synchronous oscillation of figure and ground (Fig. 2, upper diagram) as well as to figure motion alone (Fig. 2, bottom diagram) were obtained at ten different oscillation frequencies from response profiles such as shown in Fig. 1. In the experiments discussed here the oscillation amplitude amounted to $20^{\circ}$. Similar results were obtained with an oscillation amplitude of $10^{\circ}$.

With the range of oscillation frequencies used in the experiments the response to large-field motion assumes its largest amplitudes in the low frequency range between $0.0156 \mathrm{~Hz}$ and $0.0625 \mathrm{~Hz}$ (Fig. 2, upper diagram). The low frequency cut-off of the response is not yet reached at $0.0156 \mathrm{~Hz}$, the lowest frequency tested in the experiments. Response amplitudes decline steeply for oscillation
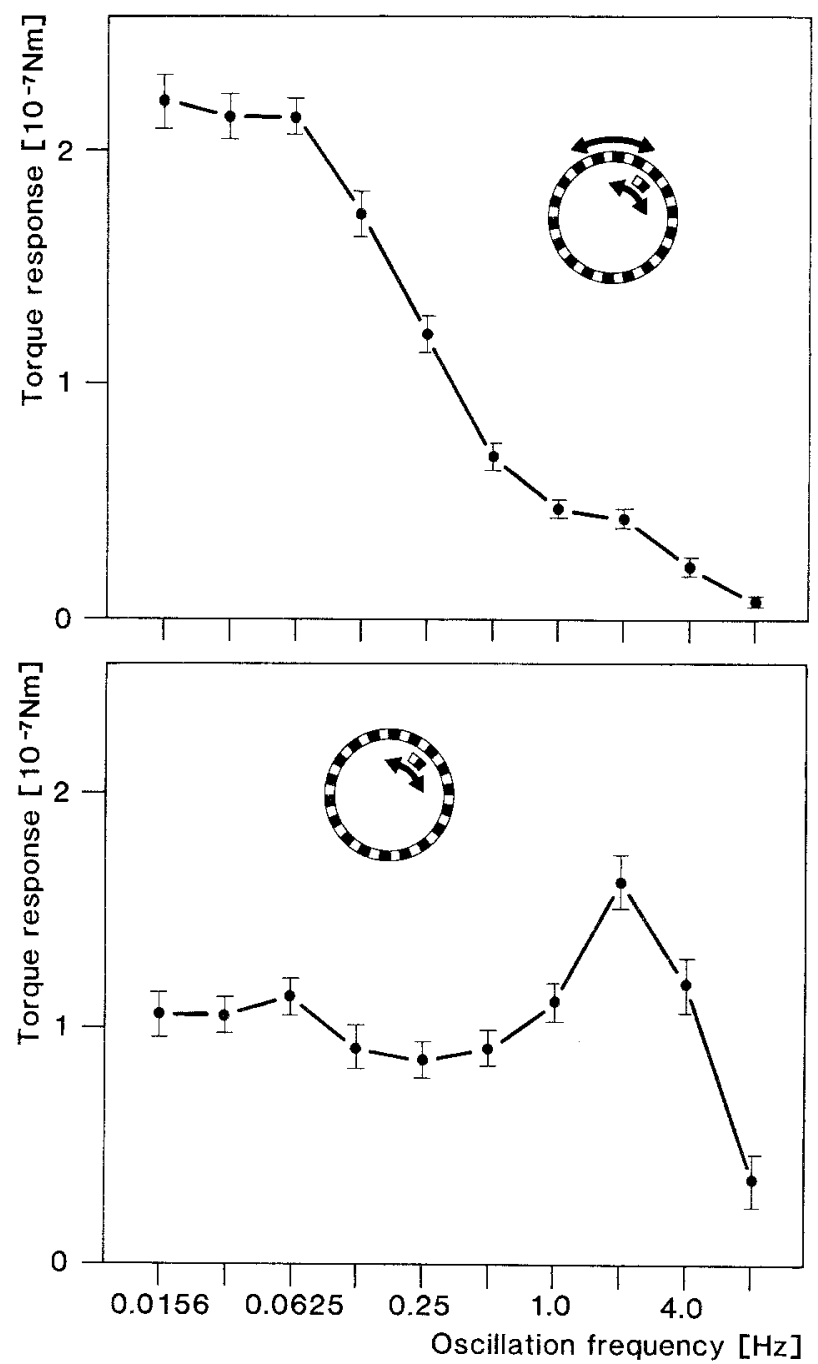

Fig. 2. Average amplitudes of the yaw torque response to synchronous oscillation of figure and ground (upper diagram) and figure motion alone (bottom diagram) as a function of oscillation frequency. Visual stimuli were same as described in the legend of Fig. 1. Experimental data were derived from averaged response profiles such as shown in Fig. 1 by evaluating the amplitudes of the responses to large-field (upper diagram) and small-field motion (bottom diagram), respectively. The data points corresponding to the different oscillation frequencies are based on measurements obtained with between 20 and 66 flies. Bars: standard error of the mean. Torque responses to largefield (upper diagram) and small-field motion (bottom diagram) depend in a different way on the oscillation frequency of the pattern. While at low frequencies the fly responds much stronger to large moving patterns than to small ones, the situation is reversed at high oscillation frequencies

frequencies greater than $0.0625 \mathrm{~Hz}$. At $2 \mathrm{~Hz}$ the amplitude is about $19 \%$ of its maximum. At $8 \mathrm{~Hz}$ the amplitude has decreased to about $4 \%$ of the maximum.

The response to a figure with a small angular horizontal extent depends in a different way on oscillation frequency (Fig. 2, bottom diagram). Under the stimulus conditions used here the re- 
sponse maximum lies at an oscillation frequency of $2 \mathrm{~Hz}$. The average response amplitude decreases towards both higher and lower oscillation frequencies. In the low frequency range it declines until about $0.25 \mathrm{~Hz}$ where it has little more than half of the maximum response amplitude. At still lower frequencies the response amplitude increases slightly again and finally reaches a kind of plateau. The final low frequency cut-off of the response amplitude is not reached within the range of oscillation frequencies used in the present study.

Thus, the responses to large-field and smallfield motion depend in quite a different way on the oscillation frequency of the stimulus pattern. At low oscillation frequencies the fly responds stronger to extended moving patterns than to small ones. In contrast, at high oscillation frequencies much larger turning amplitudes are induced by objects with a small angular horizontal extent than by wide-field motion.

\section{Discussion}

The visually induced yaw torque-response of female house-flies, as measured under open-loop conditions, depends in a specific manner on the oscillation frequency of a pattern of a particular angular size. It will be discussed in the following how these results can be related to electrophysiological studies of motion sensitive cells and how they are consistent with the performance of the fly in free flight and under artificial closed-loop conditions.

Yaw torque is controlled by two parallel systems with different spatial and temporal properties

In an earlier study (Egelhaaf 1985a-c) evidence has been accumulated that in female house-flies the compensatory optomotor turning reaction and the orientation response towards objects are mediated by two parallel motion-sensitive systems which differ in their spatial integration properties ('large-field' and 'small-field system'). The two control systems were proposed to act together in discriminating a target from its background. At the neuronal level they are likely to be represented by two functional classes of output cells of the optic lobes. While the Horizontal Cells mainly mediate information on large-field motion to the motor output (Hausen 1981, 1982; Reichardt et al. 1983), the FD-Cells are most sensitive to small moving objects (Egelhaaf 1985b). While the Horizontal- and FD-Cells of the right optic lobe are assumed to induce turns to the right, their left counterparts mediate torque responses in the opposite direction.

In the present study the dynamic properties of the yaw torque response were studied. It is concluded that not only different spatial integration properties but also different dynamic characteristics can be attributed to the two underlying control systems. Their relative contribution to the yawtorque response varies with the oscillation frequency. Hence, the previous notion (Reichardt et al. 1983) based on measurements at $2.5 \mathrm{~Hz}$ that the yaw-torque response is virtually independent of the horizontal angular extent of the stimulus pattern cannot be generalized to the entire range of oscillation frequencies.

It is suggested by the present results that at high oscillation frequencies the yaw torque is mainly controlled by the small-field system and, thus, by the FD-cells, while at low oscillation frequencies it is essentially due to the large-field system as represented by the Horizontal Cells. The latter part of this conclusion cannot solely be deduced from the present results, since at low oscillation frequencies the response to small-field motion has still about half the amplitude as the response to large-field motion. However, there is further evidence from electrophysiological experiments that at low oscillation frequencies the yaw torque is mainly controlled by the Horizontal Cells. i) The ratio of the Horizontal Cell response to small-field and large-field motion (see Reichardt et al. 1983; Egelhaaf, unpublished) is about the same as found at the behavioural level at low oscillation frequencies. ii) By relating the binocular interactions in the input circuitries of the FD- and Horizontal Cells to the behavioural responses at high and low oscillation frequencies it is suggested that the yaw-torque is controlled by these two types of visual interneurones in the way proposed above (Reichardt and Egelhaaf, in prep.).

It is not clear so far, where in the visual pathway the different frequency filtering of the two systems controlling yaw torque is accomplished. Electrophysiological results indicate, however, that the FD- and Horizontal Cells have essentially the same dynamic response properties and that the output of the Horizontal Cells is likely to be lowpass filtered in some way between the third visual ganglion and the final motor output (Egelhaaf, in prep.).

It is interesting to note that in Drosophila visually induced abdominal deflections were found which have essentially the same dynamic properties as described here for the yaw torque response to large-field motion; these rudder-like deflections 
were interpreted to assist the flight motor in course stabilization (Zanker 1987).

\section{Functional significance of the two control systems}

When an animal moves around in a structured environment, image motion over the whole retina is induced. This retinal image motion is always oppositely directed to the animal's own direction of motion. If the animal responds to these inevitable image displacements with a compensatory optomotor response, not only involuntary rotations due to external forces and internal asymmetries of the flight generating mechanism are compensated. Also intended turns of the animal towards objects might then be impeded. In the latter situation the compensatory optomotor turning reaction would be detrimental to visual orientation. In principle, this problem can be overcome by a scheme which goes back to von Helmholtz (see von Uexküll 1928), but is known as the concept of 'efference copy' (von Holst and Mittelstaedt 1950). This scheme assumes that a signal ('efference copy') is internally generated with every voluntary movement which can be used to cancel the visual feedback resulting from this movement. As a consequence, self-motion of the animal is assumed to remain unimpeded. In the following it will be argued that in case of the house-fly the different dynamic properties of the two control systems mediating yaw torque might represent a much simpler way to almost eliminate the unwanted optomotor yaw torque response to active self-motion (see also Wagner 1986b).

During free flight the performance of the fly in detecting and pursuing another moving fly is not deteriorated by a textured background. The distribution of the angle subtended by the target and the sagital plane of the pursuing fly (error angle) was found under these conditions to be virtually the same in homogeneous and textured surroundings (Wagner 1986b). This performance of the fly can be explained by relating the dynamics of retinal image motion under natural conditions to the temporal characteristics of the large-field and small-field system, respectively. During both cruising flights and pursuit manoeuvres a free-flying house-fly does not curve at an angular velocity proportional to the change of the flight path. Instead, the fly usually executes a sequence of 510 rapid turns per second which lead to retinal large-field motion of continually changing sign (Wagner 1986b). It thus appears that this retinal image motion lies within a dynamic range where the large-field optomotor system contributes to the yaw torque with only a relatively small gain. As a consequence, the fly can be assumed to stay almost unresponsive to retinal image motion induced by active self-motion of the animal. In contrast, the system which enables the fly to detect small moving objects might still control the motor output during self-motion in free flight due to its sensitivity at high oscillation frequencies. What information is actually represented by the small-field system during free flight and how this information is used to control yaw torque under natural conditions is a complicated question which cannot yet be definitely answered. This qualification, however, does not concern the above conclusion that any unwanted compensatory optomotor turning response due to active self-motion is greatly attenuated by appropriately matching the dynamic properties of the large-field system to the fly's flight dynamics.

If this were correct, one should be able to affect the error angle between the target and the tracking fly by altering the time course of the self-induced retinal background motion. In particular, under conditions of 'smooth tracking' the background does not incessantly change its direction of motion, but moves for some time in only one direction. Due to its dynamic response properties the largefield system should respond under these conditions with a compensatory yaw torque and, as a consequence, increase the error angle. As mentioned above, 'smooth tracking' does not occur in houseflies under natural conditions (Wagner 1986 b), but could be mimicked with tethered flies under artificial closed-loop conditions (Virsik and Reichardt 1976). In these experiments the tracking performance was indeed found to significantly deteriorate in the presence of a textured background just in accordance with the results of the present study.

It can, thus, be concluded that the characteristic flight dynamics and the different dynamic properties of the two systems controlling the visually guided turning behaviour of the house-fly appear to be well adapted to visual orientation under natural conditions. To eliminate the unwanted optomotor yaw torque response to self-motion the house-fly, therefore, is not required to rely upon more complicated strategies such as an efference copy scheme as has been claimed for Drosophila (Heisenberg and Wolf 1979).

In syrphid flies the situation appears to be more complicated than in house-flies (see Collett 1980). Unlike Musca, Syritta does not only show brief and rapid turns in free flight. Instead, Syritta shows smooth angular tracking, as long as the target can be kept within the fly's fovea. The large- 
field optomotor system is very likely to be activated during such tracking, such as in Musca under artificial closed-loop conditions (see above). Hence, dynamic separation of the visual consequences of active and unintended turns may not be the whole story in Syritta (Collett 1980).

The present study is only concerned with female house-flies. While there is good behavioural (Wehrhahn 1979; Wagner 1986a) and anatomical evidence (Hausen and Strausfeld 1980; Hardie et al. 1981; Hardie 1983) for a male-specific pursuit system, the large-field optomotor system is likely to have essentially the same properties in both males and females (Hausen 1987; Wehrhahn, unpublished; see also Strebel 1982). Moreover, also male house-flies do not curve smoothly but in sequences of brief turns (Wagner 1986b). Hence, the above conclusion derived from females that the large-field optomotor system does not much interfere with tracking is likely to generalize to males.

\section{Consequences}

The significance of an open-loop analysis for an understanding of visually guided behaviour has been analysed in some detail with various approaches (e.g. Reichardt and Poggio 1976; Heisenberg and Wolf 1984) but, nevertheless, is still controversial (e.g. Land 1977; Poggio 1977; Heisenberg and Wolf 1984; Wolf and Heisenberg 1986). Most likely, there will be no general solution to this problem. In accordance with previous results (see Reichardt and Poggio 1976), however, it is suggested by the present study that the knowledge of open-loop information processing, as measured in behavioural and electrophysiological experiments, can explain the performance of the housefly in certain visual orientation tasks under artificial closed-loop and even free-flight conditions. This is by no means self-evident and, therefore, substantiates the significance of this type of approach.

Acknowledgements. I am grateful to Drs. A. Borst, T. Collett, K. Hausen, W. Reichardt, H. Wagner, C. Wehrhahn, and J. Zanker for numerous suggestions and helpful discussions during the course of the experiments and for critically reading various versions of the manuscript. I also wish to thank $\mathrm{K}$. Bierig for skillful technical assistance and I. Geiss for secretarial help.

\section{References}

Bausenwein B, Wolf R, Heisenberg M (1986) Genetic dissection of optomotor behavior in Drosophila melanogaster. Studies on wild-type and the mutant optomotor-blind $d^{\mathrm{H31}}$. J Neurogenetics 3:87-109
Collett TS (1980) Angular tracking and the optomotor response. An analysis of visual reflex interaction in a hoverfly. J Comp Physiol 140:145-158

Egelhaaf M (1985a) On the neuronal basis of figure-ground discrimination by relative motion in the visual system of the fly. I. Behavioural constraints imposed on the neuronal network and the role of the optomotor system. Biol Cybern $52: 123-140$

Egelhaaf M (1985b) On the neuronal basis of figure-ground discrimination by relative motion in the visual system of the fly. II. Figure-detection cells, a new class of visual interneurones. Biol Cybern 52:195-209

Egelhaaf M (1985c) On the neuronal basis of figure-ground discrimination by relative motion in the visual system of the fly. III. Possible input circuitries and behavioural significance of the FD-cells. Biol Cybern 52:267-280

Fermi G, Reichardt W (1963) Optomotorische Reaktionen der Fliege Musca domestica. Kybernetik 2:15-28

Geiger G, Nässel DR (1982) Visual processing of moving single objects and wide-field patterns in flies: Behavioural analysis after laser-surgical removal of interneurons. Biol Cybern 44:141-149

Götz KG (1964) Optomotorische Untersuchung des visuellen Systems einiger Augenmutanten der Fruchtfliege Drosophila. Kybernetik 2:77-92

Götz KG (1983) Genetischer Abbau der visuellen Orientierung bei Drosophila. Verh Dtsch Zool Ges 76:83-99

Hardie RC (1983) Projection and connectivity of sex-specific photoreceptors in the compound eye of the male housefly (Musca domestica). Cell Tissue Res 233:1-21

Hardie RC, Franceschini N, Ribi W, Kirschfeld K (1981) Distribution and properties of sex-specific photoreceptors in the fly Musca domestica. J Comp Physiol 145:139-152

Hausen K (1981) Monocular and binocular computation of motion in the lobula plate of the fly. Verh Dtsch Zool Ges $74: 49-70$

Hausen K (1982) Motion sensitive interneurons in the optomotor system of the fly. II. The horizontal cells: Receptive field organization and response characteristics. Biol Cybern $46: 67-79$

Hausen K (1987) The neural architecture of the lobula plate of the blowfly, Calliphora erythrocephala. Cell Tissue Res (Submitted)

Hausen K, Strausfeld NJ (1980) Sexually dimorphic interneuron arrangements in the fly visual system. Proc R Soc Lond B 208:57-71

Hausen H, Wehrhahn C (1983) Microsurgical lesion of horizontal cells changes optomotor yaw responses in the blowfly Calliphora erythrocephala. Proc R Soc Lond B 219:211216

Heisenberg M, Wolf R (1979) On the fine structure of yaw torque in visual flight orientation of Drosophila melanogaster. J Comp Physiol 130:113-130

Heisenberg M, Wolf R (1984) Vision in Drosophila. Genetics of microbehavior. Springer, Berlin Heidelberg New York

Holst E von, Mittelstaedt H (1950) Das Reafferenzprinzip (Wechselwirkungen zwischen Zentralnervensystem und Peripherie). Naturwissenschaften 37:464-476

Land MF (1977) Visually guided movements in invertebrates. In: Stent GS (ed) Function and formation of neural systems. Dahlem Konferenzen, Berlin, pp 161-177

Poggio T (1977) Visually guided movements. Group report. In: Stent GS (ed) Function and formation of neural systems. Dahlem Konferenzen, Berlin, pp 309-327

Reichardt W, Poggio T (1976) Visual control of orientation behaviour in the fly. Part I: A quantitative analysis. Q Rev Biophys 9:311-375 
Reichardt W, Poggio T, Hausen K (1983) Figure-ground discrimination by relative movement in the visual system of the fly. Part II: Towards the neural circuitry. Biol Cybern [Suppl] 46:1-30

Strebel J (1982) Eigenschaften der visuell induzierten Drehmomenten-Reaktion von fixiert fliegenden Stubenfliegen Musca domestica L. und Fannia canicularis L. Dissertation, Eberhard-Karls-Universität Tübingen

Uexküll J von (1928) Theoretische Biologie. Springer, Berlin

Virsik RP, Reichardt W (1976) Detection and tracking of moving objects by the fly Musca domestica. Biol Cybern 23:83-98

Wagner H (1986a) Flight performance and visual control of flight of the free-flying housefly (Musca domestica L.) II. Pursuit of targets. Phil Trans R Soc Lond B 312:553-579

Wagner H (1986 b) Flight performance and visual control of flight of the free-flying housefly (Musca domestica L.). III Interactions between angular movement induced by wideand smallfield stimuli. Phil Trans $R$ Soc Lond B 312: $581-595$

Wehrhahn C (1979) Sex-specific differences in chasing behaviour of houseflies (Musca). Biol Cybern 32:239-241

Wehrhahn C (1985) Visual guidance of flies during flight. In: Kerkut GA, Gilbert LI (eds) Comprehensive insect physiology, biochemistry and pharmacology. Pergamon Press, Oxford New York Toronto Sydney Paris Frankfurt, pp 673684

Wolf R, Heisenberg M (1986) Visual orientation in motionblind flies is an operant behaviour. Nature 323:154-156

Zanker JM (1987) Über die Flugkrafterzeugung und Flugkraftsteuerung der Fruchtfliege Drosophila melanogaster. Dissertation, Eberhard-Karls-Universität Tübingen 